\title{
Vibration Response and Power Flow Characteristics of a Flexible Manipulator with a Moving Base
}

\author{
Yufei Liu, Wei Li, Xuefeng Yang, Mengbao Fan, Yuqiao Wang, and En Lu \\ School of Mechatronic Engineering, China University of Mining and Technology, Xuzhou 221116, China \\ Correspondence should be addressed to Wei Li; liweicumt@163.com
}

Received 9 May 2014; Revised 12 November 2014; Accepted 11 December 2014

Academic Editor: Didier Rémond

Copyright ( 2015 Yufei Liu et al. This is an open access article distributed under the Creative Commons Attribution License, which permits unrestricted use, distribution, and reproduction in any medium, provided the original work is properly cited.

\begin{abstract}
Flexible manipulator generally can be modeled as a coupling system with a flexible beam and a rigid moving base. This paper investigates the vibration responses and power flow of a flexible manipulator with a moving base (FMMB). Considering the motion characteristics of the rigid base, the moving base is modeled to have a motion with disturbances, and the dynamic model of the FMMB is established. With the dynamic model, vibration responses of the FMMB for the rigid base having disturbance velocities and accelerations are specifically presented. Subsequently, to investigate the effect of the disturbances on the vibration energy distributions of the FMMB, power flow of the FMMB is exhibited. To verify the dynamic model, an ADAMS physical model of the FMMB is constructed. It reveals that the motion characteristics of the rigid base have a noticeable effect on the vibration responses and power flow of the FMMB and should be considered. The results are significant and contribute to the vibration control of flexible manipulators.
\end{abstract}

\section{Introduction}

With the motivations of better energy efficiency, higher operation speed, and mobility, flexible manipulators have been extensively used in industrial applications, such as aerospaces and robotics fields $[1,2]$. However, due to their light weight and flexible nature, flexible manipulators exhibit vibrations during the execution of operation tasks, which significantly affects the operation precision of the end-effectors especially in high precision operating systems [3-5]. Numerous researches have investigated the vibration characteristics and control strategies of the flexible manipulators [2, 4, 6-9]. To study the vibration characteristics and devise the control strategies of a flexible manipulator, dynamic modeling should be firstly conducted. A precision dynamic model can accurately describe the vibration characteristics and contribute to the vibration control of the flexible manipulator.

As shown in Figure 1, a flexible manipulator can be modeled as a coupling system consisting of a flexible beam and a rigid base [2-4]. Driven by the rigid base, the flexible beam exhibits an elastic displacement; thus, the dynamic model is complicated by the strong coupling between the rigid base and the flexible beam [10]. In present literatures, the rigid base mainly has a translational motion or a rotational motion $[2,4,11]$; moreover, the motions velocities and accelerations of the rigid base are commonly assumed to be constant without fluctuations $[12,13]$. Actually, due to the dynamic effect of the rigid base, such as the motor parameters and mechanism inertias, this assumption is difficult to achieve and the velocities and accelerations would present a certain fluctuation [13-15]. Thus, the assumption will introduce a certain precision error in the vibration controls of the flexible manipulators. The motion characteristics of the rigid base should be considered.

In some literatures, the rigid base is assumed to exhibit a harmonic excitation, and subjected to the harmonic excitation, the beam system can be modeled as a parametrically excited system [16]. Yabuno and Nayfeh [17] investigated the nonlinear normal modes of a vertical cantilever beam excited by a principal parametric resonance. Pratiher and Dwivedy [18] studied the nonlinear vibrations of a harmonically excited single link roller-supported flexible Cartesian 


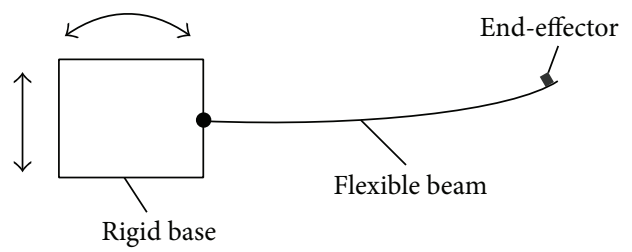

Figure 1: Schematic diagram of a flexible manipulator.

manipulator which is similar to the system presented in this paper. However, a review of these literatures can find that the harmonic excitation did not consider the motion characteristics of the rigid base, such as the velocities and the accelerations. In other words, for the rigid base having a certain motion characteristic, the effect of the harmonic disturbances has not been investigated. Moreover, the strategies to suppress the effect of the harmonic disturbances also remain a further study.

The vibration responses can describe the vibration characteristics of the end-effectors, which can be used to reflect the effect of the motion characteristics. However, the instantaneous vibration responses cannot successfully characterize the overall vibration levels of the structures. It is known that, for a vibrating structure, time-averaged vibration energy is more descriptive than instantaneous value $[19,20]$. Therefore, to further study the effect of the motion characteristics of the rigid base on the overall vibration energy distributions of the flexible manipulator, the power flow is introduced in this paper which contributes to further vibration control and structure optimization. The power flow is originally addressed by Noiseux [21] to measure uniform beams and plates and described and discussed by Goyder and White [22], which essentially combines force and velocity into a single quantity and can clearly describe the vibration energy distribution and transmission characteristics of structures. Numerous researches have focused on the power flow of beam, plate, and shell structures as well as complex coupled systems [23-27], such as the floating raft isolation systems and machine isolation systems $[28,29]$. A review of recent literatures, however, reveals that the investigations of power flow mainly focused on large-scale coupling structures or stationary flexible structures. The power flow of a moving structure, such as the flexible manipulator presented in this paper, has not obtained essential attention.

In this paper, considering the motion characteristics, the rigid base is modeled to have a motion with disturbances. The effect of the disturbances on the vibration characteristics of the FMMB is investigated for the rigid base having velocities and accelerations. To reflect the overall vibration energy of the flexible manipulator with disturbance motions, the power flow of the flexible manipulator is presented. The rest of the paper is organized as follows. In Section 2, dynamic model of the FMMB is established. Based on the dynamic model, vibration responses of the FMMB are obtained. Subsequently, vibration power flow of the FMMB is obtained in Section 3. Results are presented and discussed in Section 4. Finally, in Section 5, the paper is concluded with a brief summary.

\section{Dynamic Model and Vibration Responses of the FMMB}

In this section, dynamic model of the FMMB is established. The flexible manipulator is modeled as a coupling system consisting of a flexible beam and a rigid base as shown in Figure 2, and the flexible beam is attached to the rigid base. During the dynamic model construction, assumptions are made as follows. Motions of the flexible beam are restricted to the horizontal $x-y$ plane and transverse bending vibration is the primary motion; effect of the gravity can be neglected. Here, $s(t)$ denotes the motion of the rigid base; $w(x, t)$ represents the transverse vibration displacement of variable point $P$. Hence, the absolute displacement of $P$ can be expressed as

$$
y(x, t)=s(t)+w(x, t) .
$$

Driven by the moving base, the flexible beam presents a bending deformation. The force and moment analysis of infinitesimal element in the flexible beam is shown in Figure 3. According to the D'Alembert principle and Bernoulli-Euler Beam theory [30], the following equations can be obtained:

$$
E I \frac{\partial^{4} w(x, t)}{\partial x^{4}}+\rho A \frac{\partial^{2} y}{\partial t^{2}}=0,
$$

where $\rho$ and $A$ denote mass density and cross-sectional area of the flexible beam, respectively; $E$ is Young's modulus of the flexible beam; $I$ is the cross-sectional moment of inertia about the neural axis of the flexible beam, $I=b h^{3} / 12$, where $b$ is the width and $h$ is the thickness of the flexible beam, respectively.

Combining (1) and (2), the differential equation of transverse vibration of the FMMB can be expressed as

$$
E I \frac{\partial^{4} w(x, t)}{\partial x^{4}}+\rho A\left[\frac{\partial^{2} w(x, t)}{\partial t^{2}}+\ddot{s}(t)\right]=0,
$$

where $(\bullet)$ denotes the time derivative.

It can be obviously obtained from (3) that the motion characteristics of the moving base have an effect on the vibration characteristics of the flexible manipulator.

According to the assumed modes method [3], $w(x, t)$ can be written as

$$
w(x, t)=\sum_{i=1}^{n \rightarrow \infty} \phi_{i}(x) q_{i}(t)
$$

where $\phi_{i}(x)$ denotes the $i$ th orthogonal mode shapes and $q_{i}(t)$ denotes the $i$ th generalized coordinate. During the rigid base moving, the mode shapes of the FMMB are consistent with that of fixed-free beam. The $i$ th orthogonal mode shapes of the FMMB can be given as [30]

$$
\begin{aligned}
\phi_{i}(x) & =\sin k_{i} x-\sinh k_{i} x+\zeta\left(\cos k_{i} x-\cosh k_{i} x\right), \\
\zeta & =-\frac{\sin k_{i} x+\sinh k_{i} x}{\cos k_{i} x+\cosh k_{i} x}
\end{aligned}
$$




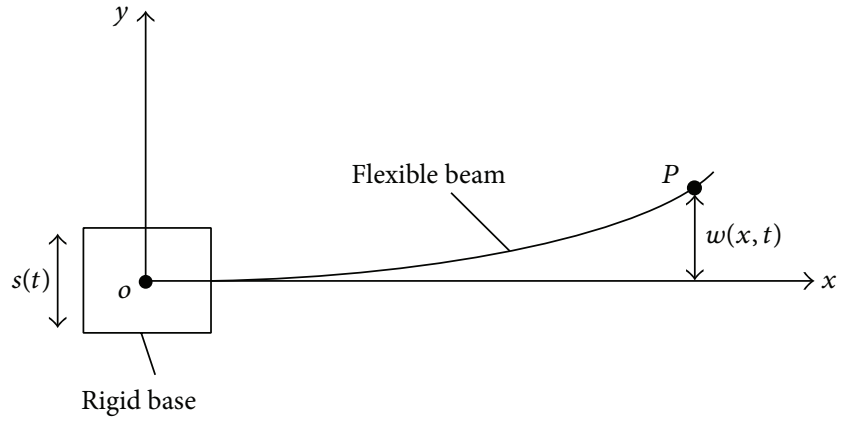

Figure 2: Dynamic model of the FMMB.

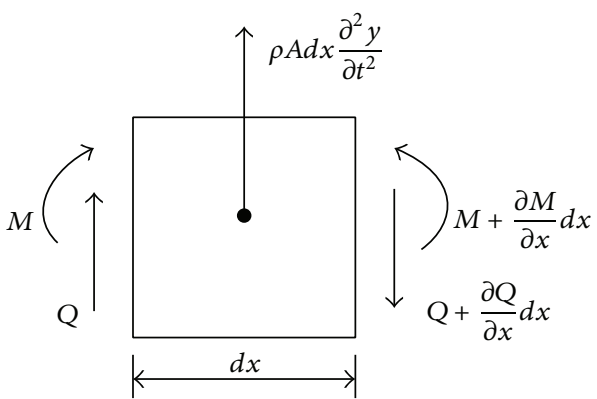

FIGURE 3: Force and moment analysis of infinitesimal element in the flexible beam.

here $k_{i}^{4}=\rho A \omega_{i}{ }^{2} / E I$, and $\omega_{i}$ is the $i$ th natural frequency of the flexible beam. The following results can be obtained:

$$
\begin{aligned}
& \frac{\partial^{4} w(x, t)}{\partial x^{4}}=\frac{\rho A}{E I} \sum_{i=1}^{n \rightarrow \infty} \omega_{i}^{2} \phi_{i}(x) q_{i}(t), \\
& \frac{\partial^{2} w(x, t)}{\partial t^{2}}=\sum_{i=1}^{n \rightarrow \infty} \phi_{i}(x) \ddot{q}_{i}(t) .
\end{aligned}
$$

Combining (6), (3) can be further written as

$$
\begin{aligned}
& \rho A \sum_{i=1}^{n \rightarrow \infty} \omega_{i}^{2} \phi_{i}(x) q_{i}(t)+\rho A\left[\sum_{i=1}^{n \rightarrow \infty} \phi_{i}(x) \ddot{q}_{i}(t)+\ddot{s}(t)\right] \\
& \quad=0 .
\end{aligned}
$$

Multiplying (7) with $\phi_{j}(x)$ and subsequently integrating the equation along the length direction, result can be obtained as

$$
\begin{aligned}
\int_{0}^{l} \phi_{j}(x)\left[\rho A \sum_{i=1}^{n \rightarrow \infty} \omega_{i}^{2} \phi_{i}(x) q_{i}(t)\right. \\
\left.+\rho A \sum_{i=1}^{n \rightarrow \infty} \phi_{i}(x) \ddot{q}_{i}(t)+\rho A \ddot{s}(t)\right] \mathrm{d} x=0 .
\end{aligned}
$$

Based on the orthogonality of mode shapes depicted as follows $[6,31]$ :

$$
\int_{0}^{l} \phi_{i}(x) \phi_{j}(x) \mathrm{d} x= \begin{cases}0, & i \neq j \\ 1, & i=j .\end{cases}
$$

Equation (8) can be expressed as

$$
\ddot{q}_{i}(t)+\omega_{i}^{2} q_{i}(t)=-\frac{m_{i}}{\rho A} \ddot{s}(t),
$$

where $m_{i}=\int_{0}^{l} \rho A \phi_{i}(x) \mathrm{d} x, i=1,2, \ldots, n$.

According to Duhamel integral [30], the solution of (10) can be obtained as follows:

$$
\begin{aligned}
q_{i}(t)= & B_{1 i} \cos \omega_{i} t+B_{2 i} \sin \omega_{i} t \\
& -\frac{1}{\omega_{i}} \int_{0}^{t} \frac{m_{i}}{\rho A} \ddot{s}(\tau) \sin \omega_{i}(t-\tau) \mathrm{d} \tau .
\end{aligned}
$$

Substituting (5) and (11) into (4), the vibration responses of the FMMB are subsequently obtained as follows:

$$
\begin{aligned}
w(x, t)=\sum_{i=1}^{n \rightarrow \infty} \phi_{i}(x)[ & B_{1 i} \cos \omega_{i} t+B_{2 i} \sin \omega_{i} t \\
& \left.-\frac{m_{i}}{\rho A \omega_{i}} \int_{0}^{t} \ddot{s}(\tau) \sin \omega_{i}(t-\tau) \mathrm{d} \tau\right],
\end{aligned}
$$

where $B_{1 i}$ and $B_{2 i}$ are determined by initial conditions and can be determined as

$$
\begin{aligned}
& B_{1 i}=\frac{1}{M_{p i}} \int_{0}^{l} \rho A w(x, 0) \phi_{i} \mathrm{~d} x, \\
& B_{2 i}=\frac{1}{\omega_{i} M_{p i}} \int_{0}^{l} \rho A \dot{w}(x, 0) \phi_{i} \mathrm{~d} x,
\end{aligned}
$$

where $M_{p i}=\int_{0}^{l} \rho A \phi_{i}{ }^{2} \mathrm{~d} x$ which is called the mode mass.

To investigate the effect of the disturbance on the vibration characteristics and simplify the analysis process, this paper mainly considers the steady-state vibration responses of the FMMB which can be expressed as follows:

$$
w(x, t)=-\sum_{i=1}^{n \rightarrow \infty} \frac{m_{i}}{\rho A \omega_{i}} \phi_{i}(x) \int_{0}^{t} \ddot{s}(\tau) \sin \omega_{i}(t-\tau) \mathrm{d} \tau .
$$

It can be obtained from (14) that, to investigate the vibration characteristics of the flexible manipulator, the motion characteristics of the rigid base should be considered. Considering the motion characteristics, the motion of the rigid base is modeled to have a disturbance which can be denoted as $f(t)$. As a function can be expanded into the form of Fourier series, to simplify the analysis process, $f(t)$ can be expressed as a sine function. Hence, the motion of the rigid base can be written as

$$
s(t)=s_{0}(t)+\gamma \sin \varepsilon t,
$$

where $s_{0}(t)$ denotes the ideal constant motion and $\gamma$ and $\varepsilon$ are the amplitude and frequency of the disturbance, respectively.

Substituting (15) into (14), results can be obtained as follows:

$$
w(x, t)=w_{0}(x, t)+w_{\gamma}(x, t)
$$


where

$$
\begin{aligned}
w_{0}(x, t)=\sum_{i=1}^{n \rightarrow \infty} \frac{m_{i}}{\rho A \omega_{i}} \phi_{i}(x) \\
\cdot\left[\dot{s}_{0}(0) \sin \omega_{i} t+s_{0}(0) \omega_{i} \cos \omega_{i} t-\omega_{i} s_{0}(t)\right. \\
\left.\quad+\omega_{i}^{2} \int_{0}^{t} s_{0}(\tau) \sin \omega_{i}(t-\tau) \mathrm{d} \tau\right], \\
w_{\gamma}(x, t)=\sum_{i=1}^{n \rightarrow \infty} \phi_{i}(x) \frac{m_{i} \gamma}{\rho A} \frac{\left(\varepsilon / \omega_{i}\right) \sin \omega_{i} t-\sin \varepsilon t}{1-\left(\omega_{i} / \varepsilon\right)^{2}} .
\end{aligned}
$$

To investigate the effect of the disturbance on the vibration responses of the FMMB, the rigid base with disturbance velocities and disturbance accelerations is presented in this paper.

For the rigid base having a disturbance velocity, the motion of the rigid base can be expressed as

$$
s(t)=v t+\gamma \sin \varepsilon t,
$$

where $v$ denotes the ideal constant velocity. According to (16), the steady-state vibration responses of the FMMB can be expressed as

$$
w(x, t)=\sum_{i=1}^{n \rightarrow \infty} \phi_{i}(x) \frac{m_{i} \gamma}{\rho A} \frac{\left(\varepsilon / \omega_{i}\right) \sin \omega_{i} t-\sin \varepsilon t}{1-\left(\omega_{i} / \varepsilon\right)^{2}} .
$$

For the rigid base having disturbance acceleration, the motion of the rigid base can be expressed as

$$
s(t)=a t^{2}+\gamma \sin \varepsilon t,
$$

where $a$ denotes the ideal constant acceleration. According to (16), the steady-state vibration responses of the FMMB can be expressed as

$$
\begin{array}{r}
w(x, t)=\sum_{i=1}^{n \rightarrow \infty} \phi_{i}(x)\left[\frac{m_{i} \gamma}{\rho A} \frac{\left(\varepsilon / \omega_{i}\right) \sin \omega_{i} t-\sin \varepsilon t}{1-\left(\omega_{i} / \varepsilon\right)^{2}}\right. \\
\left.-\frac{2 a m_{i}}{\rho A \omega_{i}{ }^{2}}\left(1-\cos \omega_{i} t\right)\right] .
\end{array}
$$

It can be obtained from (19) and (21) that the steady-state vibration responses of the FMMB are significantly influenced by the amplitude and frequency of the disturbance which will be further demonstrated in Section 4 as well as the effect of the velocities and accelerations.

\section{Vibration Power Flow of the FMMB}

To reflect the overall vibration energy of the flexible manipulator with disturbance velocities and disturbance accelerations, the power flow of the FMMB is presented in this section. As indicated in the introduction, the power flow essentially combines force and velocity into a single quantity and can clearly describe the vibration energy distribution and transmission characteristics of flexible structures. In timedomain, power flow of flexible structures can be described as [32-34]

$$
P(x)=\frac{1}{T} \int_{T}[\dot{w}(x, t) Q(x, t)+\dot{\theta}(x, t) M(x, t)] \mathrm{d} t,
$$

where $(\bullet)$ denotes the time derivative, $T=2 \pi / \omega_{i}, w$ denotes the transverse vibration displacement obtained from (14), and $Q, \theta$, and $M$ are the shear force, bending rotation, and moment of the flexible beam, respectively, and can be stated as

$$
\begin{gathered}
Q=E I \frac{\partial^{3} w}{\partial x^{3}}, \\
\theta=\frac{\partial w}{\partial x}, \\
M=E I \frac{\partial^{2} w}{\partial x^{2}} .
\end{gathered}
$$

Substituting (23) into (22), the power flow of the FMMB can be obtained as

$$
P(x)=\frac{E I}{T} \int_{0}^{T}\left[\frac{\partial^{3} w}{\partial x^{3}} \frac{\partial w}{\partial t}+\frac{\partial^{2} w}{\partial x^{2}} \frac{\partial^{2} w}{\partial x \partial t}\right] \mathrm{d} t .
$$

Considering the steady-state response, the power flow of the FMMB with a disturbance velocity and acceleration can be expressed as follows by substituting (19) and (21) into (24)

$$
\begin{aligned}
P(x)= & \frac{E I}{4 \pi \rho^{2} A^{2}} \sum_{i=1}^{n \rightarrow \infty} m_{i}^{2} \omega_{i}\left(\frac{\gamma \sin \left(2 \varepsilon / \omega_{i}\right) \pi}{1-\left(\omega_{i} / \varepsilon\right)^{2}}\right)^{2} \\
& \cdot\left(\phi_{i}^{\prime \prime \prime} \phi_{i}+\phi_{i}^{\prime \prime} \phi_{i}^{\prime}\right) .
\end{aligned}
$$

It can be obtained from (25) that the disturbances have significant effect on the power flow of the FMMB; moreover, for the steady-state responses, the power flow of the FMMB with disturbance velocities is consistent with that of the FMMB with disturbance accelerations, which will be further demonstrated in Section 4.

\section{Results and Discussions}

In order to investigate the effect of the motion characteristics on the vibration responses and power flow of the FMMB, the numerical simulation and verification are conducted in this section; also the results are presented and discussed. Properties of the flexible beam used in the numerical simulations are length $L=0.650 \mathrm{~m}$, width $b=0.050 \mathrm{~m}$, thickness $t=$ $0.002 \mathrm{~m}$, Young's modulus $E=197 \mathrm{GPa}$, volumetric density $\rho=7850 \mathrm{~kg} / \mathrm{m}^{3}$, and Poisson's ratio $\mu=0.26$. The vibration responses of the end point $(x=0.65)$ which represents the end-effector of FMMB are considered. In the assumed mode method, the first several modes are usually retained and the higher modes can be neglected [3]. Thus, only the first three modes are kept in the computations. 
Figure 4 shows the vibration responses of the FMMB for the rigid base having disturbance velocities. It indicates that the vibration responses of the FMMB with constant velocities $(\gamma=0, \varepsilon=0)$ which neglects the effects of the motion characteristics of the rigid base are consistent with that of free vibration situations, and the steady-state responses of the FMMB are unrelated with the motion velocities; this also can be obtained from (19). It clearly shows that the disturbances have a noticeable effect on the vibration responses. Specifically, considering the effect of disturbances, amplitudes of the vibration response are bigger than that of the FMMB with constant velocities $(\gamma=0, \varepsilon=0)$ which is generally considered in present literatures. The amplitudes increase with $\gamma$ and $\varepsilon$ increasing; moreover, the effect of $\varepsilon$ is more pronounced than that of $\gamma$ which can be obtained from the situations of $\gamma=0.001, \varepsilon=10$ and $=0.005, \varepsilon=2$.

The power flow of the FMMB for the rigid base having disturbance velocities is shown in Figure 5, which reflects the overall vibration energy distributions of the FMMB. It indicates that, neglecting the effects of the motion characteristics of the rigid base which is generally considered in present literatures, the power flow of the FMMB with constant velocities $(\gamma=0, \varepsilon=0)$ is consistent with that of free vibration situations. For the steady-state responses, the motion velocities have no effect on the energy distributions of the FMMB while the disturbances have a considerable effect. Considering the effect of disturbances, amplitudes of the power flow are bigger than that of the FMMB with constant velocities $(\gamma=0, \varepsilon=0)$. The amplitudes increase with $\gamma$ and $\varepsilon$ increasing; moreover, compared with $\gamma$, the effect of $\varepsilon$ is more pronounced which can be indicated in the situations of $\gamma=0.001, \varepsilon=10$ and $\gamma=0.005$, and $\varepsilon=2$ which is similar to the vibration responses characteristics.

Figure 6 presents the vibration responses of the FMMB for the rigid base having disturbance accelerations. It can be obtained that the disturbances have a noticeable effect on the vibration responses. Specifically, amplitudes of the vibration responses are bigger than that of the FMMB with constant accelerations $(\gamma=0, \varepsilon=0)$ which is generally considered in present literatures. Similar to the FMMB with disturbance velocities, the amplitudes increase with $\gamma$ and $\varepsilon$ increasing, and $\varepsilon$ has a more considerable effect which can be indicated in the situations of $\gamma=0.001, \varepsilon=10$ and $=0.005$, $\varepsilon=2$. Moreover, it can be obtained from Figure 6 that, with the accelerations increasing, the amplitudes increased while the effect of the disturbances on the vibration responses decreased; at the same time, the vibration responses gradually approach the FMMB with constant accelerations $(\gamma=0, \varepsilon=$ 0 ). This further reveals that larger accelerations contribute to suppressing the effect of the disturbances. This is significant for further vibration controls and optimizations of the motion characteristics of the rigid base.

The power flow of the FMMB for the rigid base having disturbance accelerations is shown in Figure 7. It can be obviously obtained that, for the steady-state responses, the power flow characteristics are consistent with that of the FMMB with disturbance velocities as shown in Figure 5. This further indicates that the power flow is unrelated with the motion velocities and accelerations which also can be

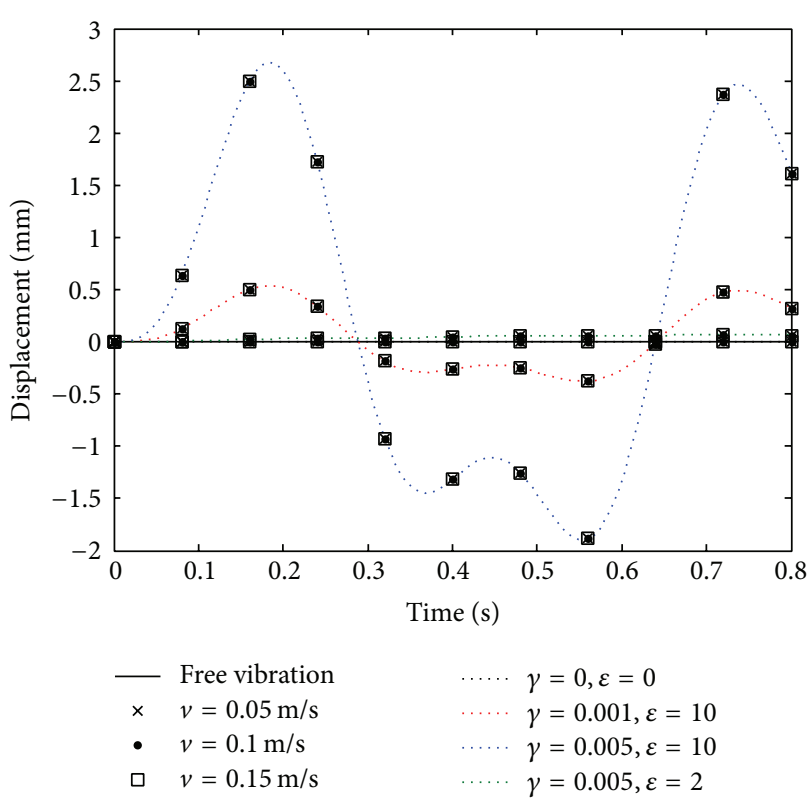

FIGURE 4: Vibration responses of the FMMB with disturbance velocities.

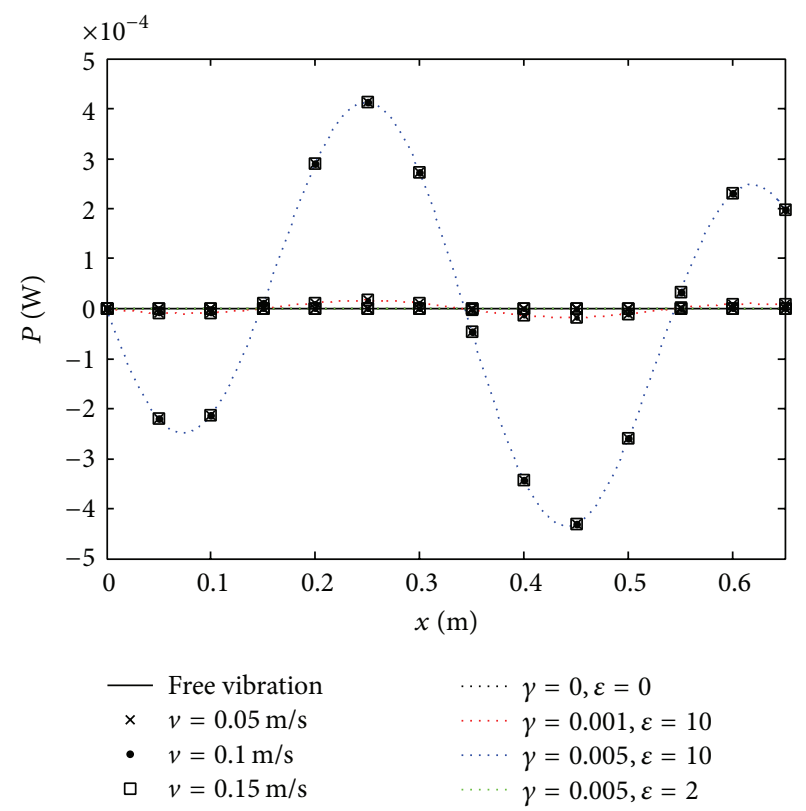

Figure 5: Power flow of the FMMB with disturbance velocities.

obtained from (25). Similar conclusion can be obtained that the disturbances have a noticeable effect on the power flow. Amplitudes of the power flow are bigger than that of the FMMB with constant accelerations $(\gamma=0, \varepsilon=0)$ and increase with $\gamma$ and $\varepsilon$ increasing. Moreover, the effect of $\varepsilon$ is more pronounced which can be obtained from the situations of $\gamma=0.001, \varepsilon=10$ and $=0.005, \varepsilon=2$.

To verify the dynamic model and numerical results, an ADAMS physical model of the FMMB is constructed. The properties of the flexible beam used in the physical model are consistent with that of the numerical simulations. 

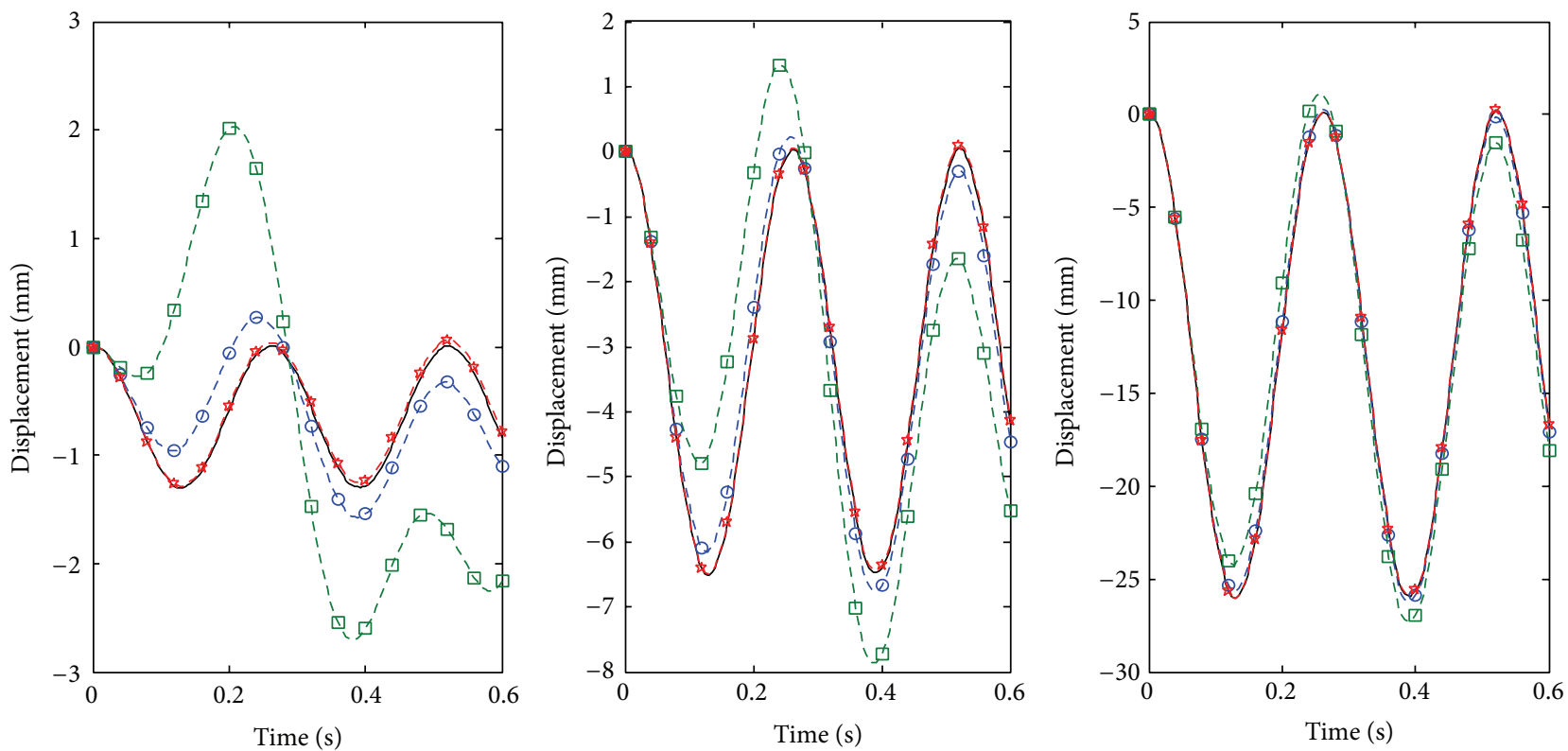

$$
\begin{aligned}
& -a=0.1(\gamma=0, \varepsilon=0) \\
& -\ominus-\gamma=0.001, \varepsilon=10 \\
& \text { 一曰- } \gamma=0.005, \varepsilon=10 \\
& \text { - } \gamma=0.005, \varepsilon=2
\end{aligned}
$$

$$
\begin{aligned}
& -a=0.5(\gamma=0, \varepsilon=0) \\
& -\ominus-\gamma=0.001, \varepsilon=10 \\
& \text {-曰- } \gamma=0.005, \varepsilon=10 \\
& \text { 一罡- } \gamma=0.005, \varepsilon=2
\end{aligned}
$$

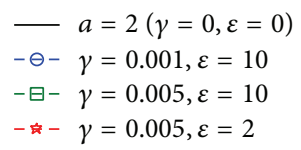

FIgURE 6: Vibration responses of the FMMB with disturbance accelerations.

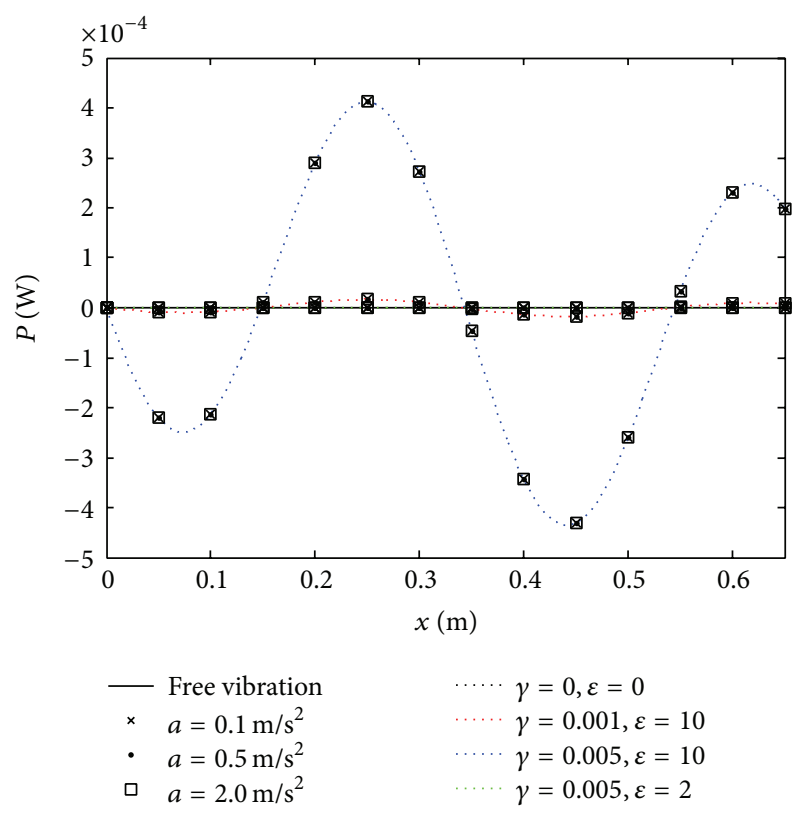

FIGURE 7: Power flow of the FMMB with disturbance accelerations.

The vibration responses of the FMMB for the rigid base having disturbance accelerations $\left(a=0.5 \mathrm{~m} / \mathrm{s}^{2}\right)$ are shown in Figure 8. It can be obtained that the numerical results presented in this paper are well consistent with the results of the physical model. It should be noted that, due to the fact that the dynamic model did not consider the structural damping

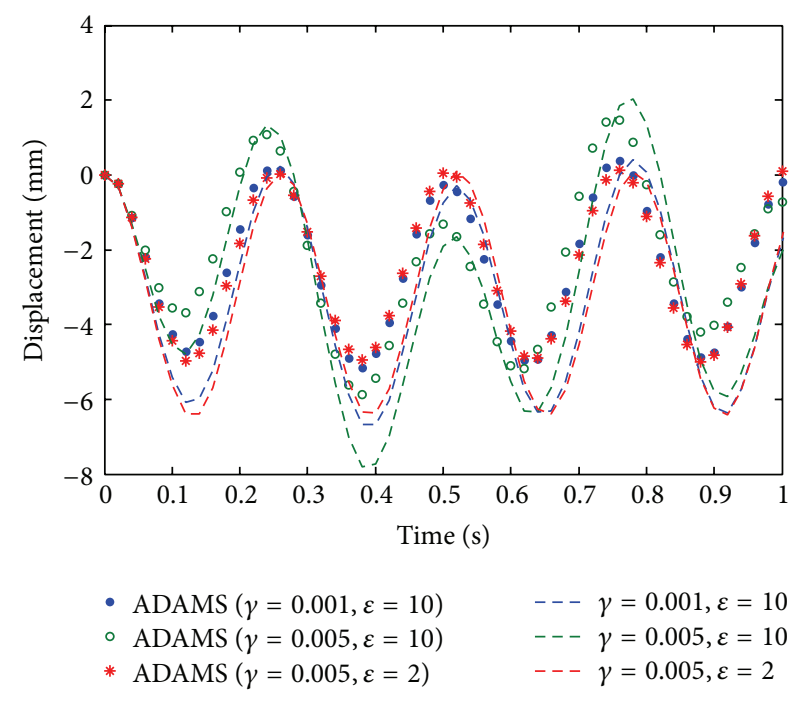

FIGURE 8: Numerical and experimental vibration responses of the $\operatorname{FMMB}\left(a=0.5 \mathrm{~m} / \mathrm{s}^{2}\right)$.

which actually exists in the physical model, the numerical amplitudes are bigger than that of the physical model while the change trends are well consistent.

\section{Conclusions}

Considering the motion characteristics of the rigid base, the rigid base is modeled to have a motion with disturbances. 
The effect of the disturbances on the vibration responses and power flow of the FMMB is investigated. It demonstrated that the motion characteristics of the rigid base have a noticeable effect on the vibration responses and power flow. Specifically, considering the effect of disturbances of the rigid base, amplitudes of the vibration responses and power flow are bigger than that of FMMB with ideal constant velocities and accelerations. Furthermore, the amplitudes increase with disturbance frequencies and disturbance amplitudes increasing, and the effect of disturbance frequencies is more pronounced. For the steady-state responses, the velocities and accelerations have no effect on the power flow of the FMMB. The vibration responses are unrelated with the velocities while the accelerations have a noticeable effect on the vibration responses. With the accelerations increasing, the amplitudes increased while the effect of the disturbances on the vibration responses decreased; at the same time, the vibration responses gradually approach the FMMB with constant accelerations. This further reveals that larger accelerations can suppress the effect of the disturbances of the rigid base. The results are significant for the vibration controls and structure optimization of flexible manipulators.

\section{Conflict of Interests}

The authors declare that there is no conflict of interests regarding the publication of this paper.

\section{Acknowledgments}

This research work was partially supported by the National Natural Science Foundation of China (no. 51305444), the Doctoral Fund of Ministry of Education under Grant (no. 20120095120013), the Scientific and Technological Project of Jiangsu Province (BY2014028-06), the Postgraduate Cultivation and Innovation Project of Jiangsu Province (KYLX_1375), and the project funded by the Priority Academic Program Development of Jiangsu Higher Education Institutions (PAPD). The authors sincerely thank the reviewers for their constructive comments and suggestions which made substantial improvements to this paper.

\section{References}

[1] S. S. Ge, T. H. Lee, and G. Zhu, "Asymptotically stable end-point regulation of a flexible SCARA/Cartesian robot," IEEE/ASME Transactions on Mechatronics, vol. 3, no. 2, pp. 138-144, 1998.

[2] M. Dadfarnia, N. Jalili, Z. Liu, and D. M. Dawson, "An observerbased piezoelectric control of flexible Cartesian robot arms: theory and experiment," Control Engineering Practice, vol. 12, no. 8, pp. 1041-1053, 2004.

[3] S. K. Dwivedy and P. Eberhard, "Dynamic analysis of flexible manipulators, a literature review," Mechanism and Machine Theory, vol. 41, no. 7, pp. 749-777, 2006.

[4] Z.-C. Qiu, "Adaptive nonlinear vibration control of a Cartesian flexible manipulator driven by a ballscrew mechanism," Mechanical Systems and Signal Processing, vol. 30, pp. 248-266, 2012.
[5] M. A. Neto, J. A. Ambrósio, L. M. Roseiro, A. Amaro, and C. M. Vasques, "Active vibration control of spatial flexible multibody systems," Multibody System Dynamics, vol. 30, no. 1, pp. 13-35, 2013.

[6] S. S. Ge, T. H. Lee, and J. Q. Gong, "A robust distributed controller of a single-link SCARA/Cartesian smart materials robot," Mechatronics, vol. 9, no. 1, pp. 65-93, 1999.

[7] M. Dadfarnla, N. Jalili, B. Xian, and D. M. Dawson, "A lyapunov-based piezoelectric controller for flexible cartesian robot manipulators," Journal of Dynamic Systems, Measurement and Control, Transactions of the ASME, vol. 126, no. 2, pp. 347358, 2004.

[8] C. M. A. Vasques and J. Dias Rodrigues, "Active vibration control of smart piezoelectric beams: comparison of classical and optimal feedback control strategies," Computers and Structures, vol. 84, no. 22-23, pp. 1402-1414, 2006.

[9] K. Gurses, B. J. Buckham, and E. J. Park, "Vibration control of a single-link flexible manipulator using an array of fiber optic curvature sensors and PZT actuators," Mechatronics, vol. 19, no. 2, pp. 167-177, 2009.

[10] W. Chen, "Dynamic modeling of multi-link flexible robotic manipulators," Computers \& Structures, vol. 79, no. 2, pp. 183195, 2001.

[11] J. B. Yang, L. J. Jiang, and D. C. Chen, "Dynamic modelling and control of a rotating Euler-Bernoulli beam," Journal of Sound and Vibration, vol. 274, no. 3-5, pp. 863-875, 2004.

[12] K. Wei, G. Meng, S. Zhou, and J. Liu, "Vibration control of variable speed/acceleration rotating beams using smart materials," Journal of Sound and Vibration, vol. 298, no. 4-5, pp. 1150-1158, 2006.

[13] Z. Li, G. Cai, Q. Huang, and S. Liu, "Analysis of nonlinear vibration of a motor-linkage mechanism system with composite links," Journal of Sound and Vibration, vol. 311, no. 3-5, pp. 924940, 2008.

[14] F. W. Liou, A. G. Erdman, and C. S. Lin, "Dynamic analysis of a motor-gear-mechanism system," Mechanism and Machine Theory, vol. 26, no. 3, pp. 239-252, 1991.

[15] U. Andreaus and P. Casini, "Dynamics of friction oscillators excited by a moving base and/or driving force," Journal of Sound and Vibration, vol. 245, no. 4, pp. 685-699, 2001.

[16] A. H. Nayfeh and D. T. Mook, Nonlinear Oscillations, Wiley, Victoria, Canada, 1995.

[17] H. Yabuno and A. H. Nayfeh, "Nonlinear normal modes of a parametrically excited cantilever beam," Nonlinear Dynamics, vol. 25, no. 1-3, pp. 65-77, 2001.

[18] B. Pratiher and S. K. Dwivedy, "Non-linear dynamics of a flexible single link Cartesian manipulator," International Journal of Non-Linear Mechanics, vol. 42, no. 9, pp. 1062-1073, 2007.

[19] X. Xue, G. Li, Y. Xiong, and J. Gong, "Power flow response based dynamic topology optimization of bi-material plate structures," Chinese Journal of Mechanical Engineering, vol. 26, no. 3, pp. 620-628, 2013.

[20] Y. P. Xiong, J. T. Xing, and W. G. Price, "A general linear mathematical model of power flow analysis and control for integrated structure-control systems," Journal of Sound and Vibration, vol. 267, no. 2, pp. 301-334, 2003.

[21] D. U. Noiseux, "Measurement of power flow in uniform beams and plates," The Journal of the Acoustical Society of America, vol. 47, no. 1, pp. 238-247, 1970.

[22] H. G. D. Goyder and R. G. White, "Vibrational power flow from machines into built-up structures, part I: introduction 
and approximate analyses of beam and plate-like foundations," Journal of Sound and Vibration, vol. 68, no. 1, pp. 59-75, 1980.

[23] T. Y. Li, T. Zhang, J. X. Liu, and W. H. Zhang, "Vibrational wave analysis of infinite damaged beams using structure-borne power flow," Applied Acoustics, vol. 65, no. 1, pp. 91-100, 2004.

[24] J. M. Cuschieri, "Structural power-flow analysis using a mobility approach of an L-shaped plate," Journal of the Acoustical Society of America, vol. 87, no. 3, pp. 1159-1165, 1990.

[25] J. Yan, T. Y. Li, J. X. Liu, and X. Zhu, "Input power flow in a submerged infinite cylindrical shell with doubly periodic supports," Applied Acoustics, vol. 69, no. 8, pp. 681-690, 2008.

[26] G. P. Feng, Z. Y. Zhang, Y. Chen, and H. X. Hua, "Research on transmission paths of a coupled beam-cylindrical shell system by power flow analysis," Journal of Mechanical Science and Technology, vol. 23, no. 8, pp. 2138-2148, 2009.

[27] Y. P. Xiong, J. T. Xing, and W. G. Price, "Power flow analysis of complex coupled systems by progressive approaches," Journal of Sound and Vibration, vol. 239, no. 2, pp. 275-295, 2001.

[28] W. J. Choi, Y. P. Xiong, and R. A. Shenoi, "Power flow analysis for a floating sandwich raft isolation system using a higher-order theory," Journal of Sound and Vibration, vol. 319, no. 1-2, pp. 228246, 2009.

[29] X. Ma, G. Jin, and Z. Liu, "Active structural acoustic control of an elastic cylindrical shell coupled to a two-stage vibration isolation system," International Journal of Mechanical Sciences, vol. 79, pp. 182-194, 2014.

[30] S. R. Singiresu, Mechanical Vibration, Pearson Education, 4th edition, 2004.

[31] S. O. R. Moheimani and A. J. Fleming, Fundamentals of Piezoelectricity. Piezoelectric Transducers for Vibration Control and Damping, Springer, London, UK, 2006.

[32] X. Q. Wang, W. O. Wong, and L. Cheng, "Modal power flow with application to damage detection," International Journal of Engineering Science, vol. 47, no. 4, pp. 512-523, 2009.

[33] S. J. Walsh and R. G. White, "Measurement of vibrational power transmission in curved beams," Journal of Sound and Vibration, vol. 241, no. 2, pp. 157-183, 2001.

[34] S. X. Wang, Z. Jiang, and Z. W. Zhu, "A new method for measuring power flow of one-dimensional vibrating structure," Journal of Vibrationengineering, vol. 16, no. 3, pp. 368-372, 2003. 

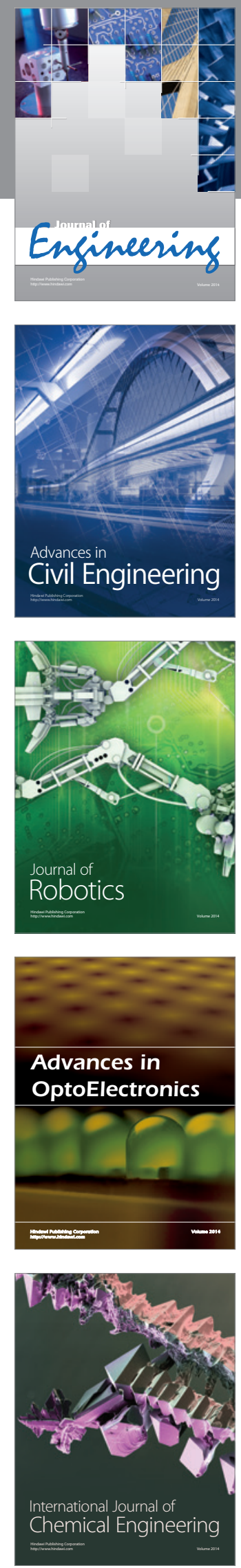

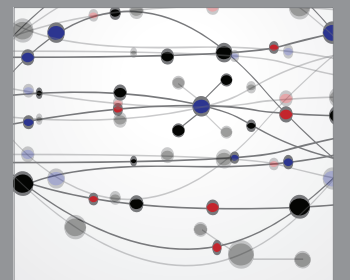

The Scientific World Journal
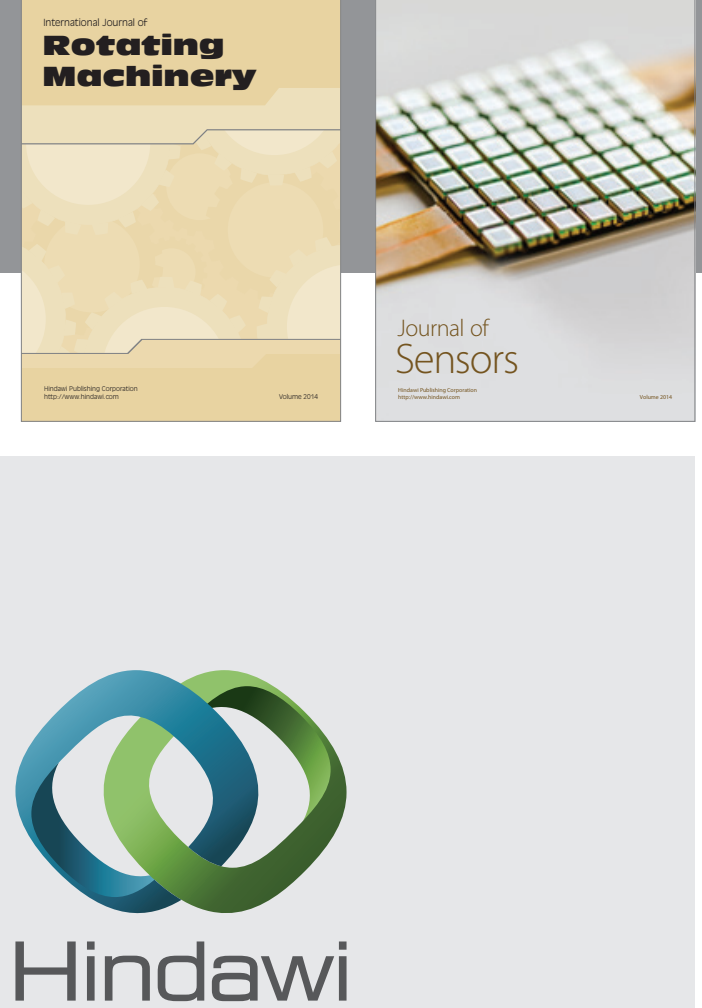

Submit your manuscripts at http://www.hindawi.com
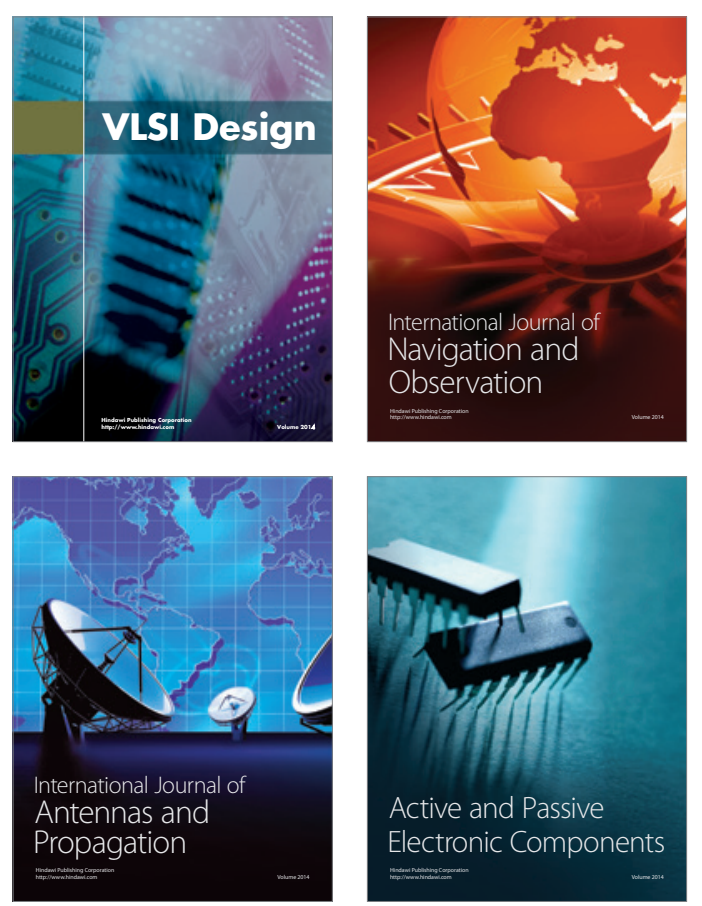
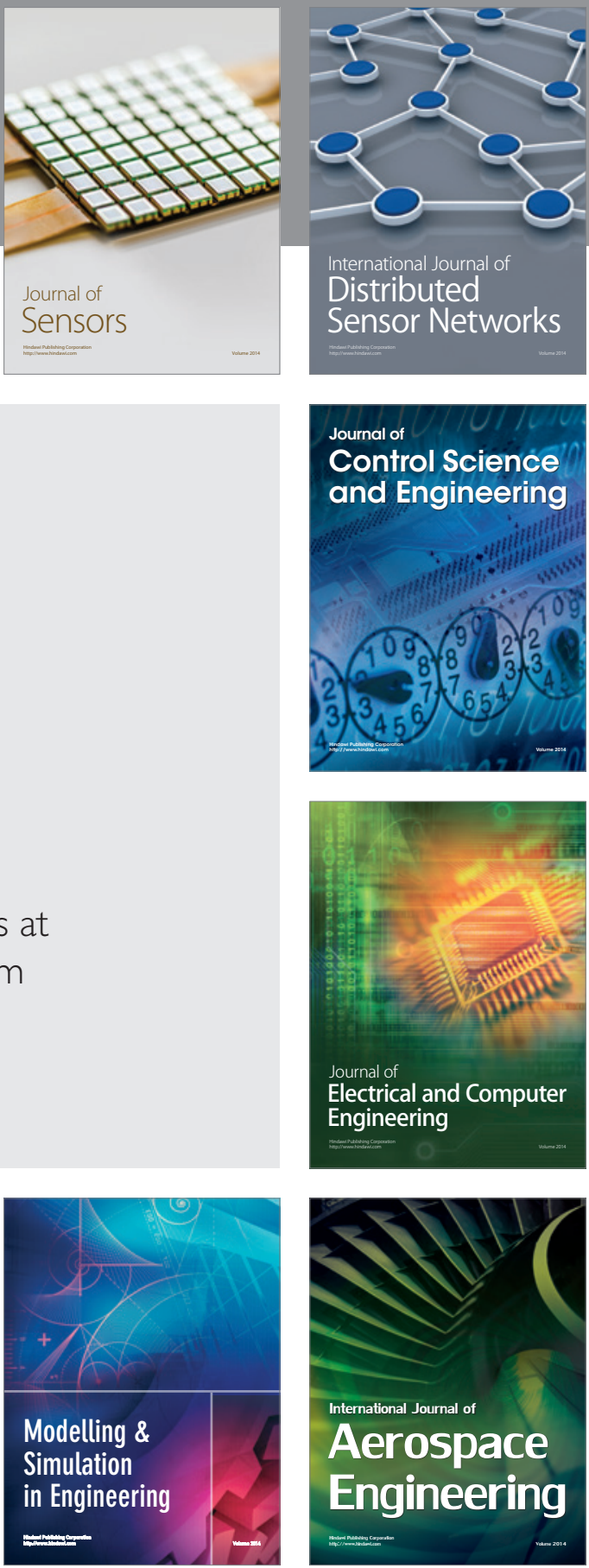

Journal of

Control Science

and Engineering
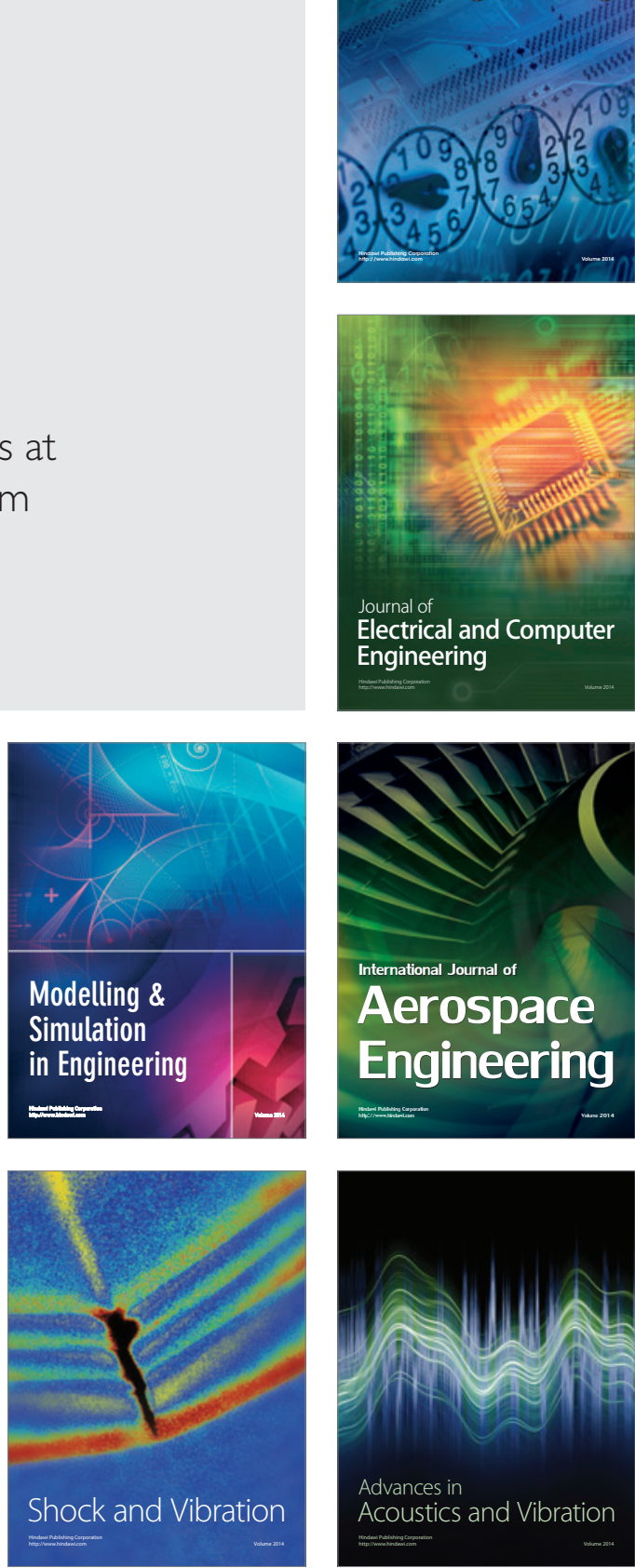